J. Lake Sci. (湖泊科学), 2018, 30(5): 1379-1387

DOI 10. 18307/2018. 0519

(c) 2018 by Journal of Lake Sciences

\title{
福寿螺入侵中国的扩散动态及潜在分布”
}

\author{
周 宇 ${ }^{1}$, 袁雪颖 ${ }^{1}$, 杨子轩 $^{1}$, 刘雄军 ${ }^{2}$, 欧阳珊 ${ }^{1}$, 吴小平 ${ }^{1 * *}$ \\ (1: 南昌大学生命科学学院, 南昌 330031) \\ (2: 南昌大学鄱阳湖环境与资源利用教育部重点实验室, 南昌 330031)
}

\begin{abstract}
摘 要: 福寿螺 (Pomacea spp.) 严重破坏了农业生产和生态系统,并且对人类健康形成致命的威胁. 其中小管福寿螺 (Pomacea canaliculata) 是世界 100 种恶性外来人侵物种之一. 通过收集福寿螺分布数据绘制分布动态图, 重建福寿螺在 我国的扩散动态, 明晰福寿螺的扩散方向和扩散速度. 扩散过程表明福寿螺在中国南方的分布区将会继续扩大范围,有 进一步向北扩散的趋势, 扩散速度持续增长, 未来可能在南方形成全面人侵格局. 使用气候和海拔数据变量构建最大熵 模型, 预测福寿螺的潜在分布区和影响福寿螺分布的关键环境变量, 结果显示: 浙江、福建、江西、广东、广西、海南和台湾 等省已成为福寿螺的高分布区, 其危害十分严重; 上海、湖北、湖南、四川、西藏、贵州、重庆和云南为中分布区, 但也具有 较高的潜在暴发风险. 环境变量分析显示: 年均温度是影响福寿螺分布的最重要环境变量. 受试者工作曲线 ( ROC) 检测 了模型预测结果的准确度, AUC 平均值为 0.97 , 说明预测结果准确, 可信度高. 这些结果可为进一步开展福寿螺在我国的 扩散趋势预测、建立风险评估体系以及制定有效的防治措施提供依据.
\end{abstract}

关键词：Maxent 模型;福寿螺;潜在分布;扩散动态

\section{Spread pattern and potential distribution of the invasive species Pomacea spp. in China}

\author{
ZHOU Yu ${ }^{1}$, YUAN Xueying ${ }^{1}$, YANG Zixuan $^{1}$, LIU Xiongjun ${ }^{2}$, OUYANG Shan ${ }^{1} \&$ WU Xiaoping ${ }^{1 * *}$ \\ (1: School of Life Sciences, Nanchang University, Nanchang 330031, P.R. China) \\ (2: Key Laboratory of Poyang Lake Environment and Resource Utilization, Ministry of Education, Nanchang University, Nan- \\ chang 330031, P.R.China)
}

Abstract: Pomacea spp. have caused negative impact on agricultural production, ecological environment and potential human health pests. Pomacea canaliculata is one of the 100 worst invasive alien species in the world. Distribution data of Pomacea spp. was collected for reconstruction of the spread history of Pomacea spp. in China. The expansion process showed that the distribution of Pomacea spp. in southern China would continue to expand, and a comprehensive invasion pattern may be formed in the future with a tendency to further spread to the North. Combined with the climate and altitude data, Maxent model was constructed to predict the potential distribution of Pomacea spp. and analyzed the key environmental factors affecting its distribution. The results showed that Zhejiang, Fujian, Jiangxi, Guangdong, Guangxi, Hainan and Taiwan had become a high distribution areas with serious damages, and Shanghai, Hubei, Hunan, Sichuan, Xizang, Guizhou and Yunnan were in medium distribution, but with a high risk for outbreaks. Analysis of environmental variables showed that the influence of annual mean temperature was the most critical. The receiver operator characteristic curve (ROC) was used to test the predicted results, with the average AUC value of 0.97 which indicated a high prediction accuracy and credible results. These results could provide the basis for further developing the prediction of dispersal tendency of Pomacea spp. in China, establishing the risk assessment system and formulating effective prevention policy. Keywords: Maxent model; Pomacea spp.; potential distribution; spread pattern

生物人侵已成为 21 世纪 5 大全球性重要环境问题之一, 它给人侵地区的生物多样性、生态系统平衡、社 会经济和人类健康造成十分严重的危害 ${ }^{[1]}$. 近年来, 我国外来人侵物种数量达 283 种之多, 造成的损失高达

* 国家重点研发计划项目 (2016YFC1202002) 资助. 2017-12-04 收稿;2018-01-07 收修改稿. 周宇( 1994 ), 男, 硕 士研究生;E-mail: 1151556864@qq.com.

** 通信作者;E-mail: xpwu@ ncu.edu.cn. 
1198.76 亿元 ${ }^{[2]}$. 福寿螺 (Pomacea spp.) 隶属软体动物门 (Mollusca)、腹足纲 (Gastropoda)、瓶螺科 (Pilaidae)、 福寿螺属 (Pomacea), 又称大瓶螺或金苹果螺, 原产于南美洲亚马逊河流域, 目前已人侵北美洲、非洲、欧洲、 东南亚等地区, 其中小管福寿螺 (Pomacea canaliculata) 已成为世界 100 种恶性外来人侵物种之一 ${ }^{[3]}$. 福寿螺 不仅啃食农作物, 危害农业生产, 还侵占了本地螺类的生态位, 对生态系统完整性和当地经济造成严重的破 坏 ${ }^{[4-5]}$. 同时福寿螺是广州管圆线虫重要的中间寄主之一, 给人类健康带来潜在威胁 ${ }^{[6]}, 2003$ 年福寿螺被列 人首批人侵中国的 16 种“危害最大的外来物种”名单 ${ }^{[7]}$.

目前关于福寿螺形态特征 ${ }^{[8-9]}$ 、繁殖特性 ${ }^{[10-11]}$ 、养殖技术 ${ }^{[12]}$ 以及综合防治 ${ }^{[13-14]}$ 的研究较多, 而对福寿螺 的分布研究多为小尺度, 例如刘雨芳等 ${ }^{[15]}$ 研究湖南省福寿螺分布现状, 绘制了福寿螺在湖南的分布现状图 与扩散风险预警图. 何铭谦等 ${ }^{[16]}$ 基于 GIS 技术对福寿螺在广东地区的适宜生境进行划分. 为了预测物种在 大尺度区域的潜在分布,生态学家常用方法是构建生态位模型 ${ }^{[17]}$. Elith 等 ${ }^{[18]}$ 基于 16 种生态位模型对 226 个物种的适生区预测效果进行比较研究, 认为模型类型选择与物种属性具有相关性. 广泛用于预测物种分 布的生态位模型主要有 BIOCLIM DOMAIN、GARP 和 Maxent, 但每个生态位模型的适用性均不一样. 张海涛 等 ${ }^{[19]}$ 应用 4 个生态位模型对人侵物种福寿螺进行预测, 指出 Maxent 模型相对于其他模型是预测效果最好 的. 该模型应用于预测蓄杆双脐螺 ${ }^{[20]}$ 、三旋丽卷螺 ${ }^{[21]}$ 、稻水象甲 ${ }^{[22]}$ 、松材线虫 ${ }^{[23]}$ 、麦穗鱼和鲫鱼 ${ }^{[24]}$ 等外来 人侵物种的潜在分布区,其结果均能较好地反映物种的实际分布 ${ }^{[25]}$. Byers 等 ${ }^{[26]}$ 基于 Maxent 预测福寿螺在 美国东南部的潜在分布, 证明气候和环境变量构建的生态位模型是预测人侵物种分布强有力的方法. 张海 涛等 ${ }^{[19]}$ 使用 337 条福寿螺分布数据预测在中国的适生区, 但由于样本搜集分布数据较少, 缺少海拔数据, 可 能对预测结果的可靠性有所影响. 基于此, 本研究从时间尺度上评估福寿螺在中国的分布概况和扩散速度, 并基于 Maxent 模型预测福寿螺在中国的潜在分布, 分析影响福寿螺潜在分布的主要环境变量, 以期对未来 可能人侵的的地区提出预警.

\section{1 材料与方法}

\section{1 材料}

1.1.1 分布数据 福寿螺分布数据来源: ( 1) 中国外来人侵生物数据库 (http://www. chinaias.cn) $)^{[27]} ;$ ( 2) 2006- 2007 年全国广州管圆线虫病自然疫源地流行病学调查的采样记录 ${ }^{[28]}$; (3) 1985 年以来公开发表的 学术论文以及统计年鉴; (4) 野外实地调查. 获得的分布数据用 Google Earth 核实坐标信息, 去除重复和采 集地点不准确的分布数据, 最后获得 1981-2017 年全国有效分布数据 683 条. 由于福寿螺的适应性极强,所 以一旦出现人侵记录即视为该地区至今仍有分布, 并假定文献记录时间或者数据库记录时间以及实地调查 时间为其最早人侵的时间 ${ }^{[29]}$, 根据福寿螺最早的人侵记录, 每 10 年统计福寿螺分布数据, $1980 \mathrm{~s}$ 分布点数据 有 22 条, 1990 s 有 72 条, 2000s 有 380 条,2017 年有 683 条,数据集分别保存为 $\operatorname{shp}$ 和 csv 格式.

1.1.2 环境图层数据 从世界气候数据库 Worldclim (http://www.worldclim.org/) 上下载分辨率为 $30^{\prime \prime}(1 \mathrm{~km} \times$ $1 \mathrm{~km}$ ) 的环境变量图层, 包括 19 个生物气候变量和一个海拔变量图层 (表 1). 根据外来人侵数据库的记录 和 2006- 2007 年全国广州管圆线虫病自然疫源地流行病学调查的采样记录范围, 本研究利用 ArcGIS 软件 中的 Extract 工具从世界环境图层中提取由 $39^{\circ} \mathrm{E}$ 至我国东部海域, $53^{\circ} \mathrm{N}$ 至我国台湾南部海域的环境图层数 据用于后续分析.

由于环境变量之间可能存在相关性,利用 ArcGIS 的空间分析工具对 20 个环境变量进行空间自相关分 析 ${ }^{[30]}$, 若相关系数 $|r| \geqslant 0.8$, 只能选取其中的一个变量, 最终得到 6 个环境变量图层 (Bio1、Bio3、Bio4、 Bio12、Bio15 和 Bio20), ArcGIS 软件中的 Conversion 工具将其转化成 ASC II 格式, 建立最优模型.

\section{2 方法}

1.2.1 不同年代的扩散速度 由于经济、人文和地理等因素会使人侵种在某些特定方向上的扩散受到限制, 从而使人侵种的分布区呈现不规则的形状 ${ }^{[31-32]}$. 因此我们采用邻里测量法计算福寿螺的扩散速度, 其计算 公式为 $\Delta r=\left[\left(\Delta r_{\text {max }}^{2}+\Delta r_{\text {min }}^{2}\right) / 2\right]^{1 / 2}, \Delta r_{\text {max }}$ 和 $\Delta r_{\text {min }}$ 分别表示分布区原地理边缘分布点和新地理边缘分布点之间 的最长和最短距离,各邻里间的扩散速度累积形成总的扩散 ${ }^{[33-34]}$. 
表 1 环境图层的数据变量

Tab.1 Environmental variables in layers

\begin{tabular}{llll}
\hline 变量 & 描述 & 变量 & 描述 \\
\hline Bio1 & 年均温 & Bio11 & 最冷季度平均温度 \\
Bio2 & 昼夜温差月均值 & Bio12 & 年均降水量 \\
Bio3 & 等温性 & Bio13 & 最湿月降水量 \\
Bio4 & 温度季节性变化标准差 & Bio14 & 最干月降水量 \\
Bio5 & 最暖月最高温度 & Bio15 & 降水量季节性变异系数 \\
Bio6 & 最冷月最低温度 & Bio16 & 最湿季度降水量 \\
Bio7 & 年均气温变化范围 & Bio17 & 最干季度降水量 \\
Bio8 & 最湿季度平均温度 & Bio18 & 最暖季度降水量 \\
Bio9 & 最干季度平均温度 & Bio19 & 最冷季度降水量 \\
Bio10 & 最暖季度平均温度 & Bio20 & 海拔 \\
\hline
\end{tabular}

1.2.2 Maxent 模型分析 将相应格式的环境图层数据和福寿螺分布数据集导人 Maxent 软件,构建 Maxent 模 型. Maxent 模型参数设置: 最大迭代次数为 2000 , Bootstrap 重复运算 10 次, 开启 Random seed, 选择刀切法 (Jackknife) 分析主要环境变量. 将 $80 \%$ 的福寿螺分布数据用于模型构建和预测, $20 \%$ 用于模型验证. 勾选 ROC 曲线选项绘制 ROC 曲线并计算曲线下面积 $\mathrm{AUC}$ 来评估模型的拟合效果, 若 $\mathrm{AUC}<0.6$, 模型预测效果 很差; 若 $0.6<\mathrm{AUC}<0.9$, 模型预测效果中等; 若 $\mathrm{AUC}>0.9$, 模型预测效果很好 ${ }^{[35-36]}$. Maxent 模型分析需调整正 则化乘数 (regularization multiplier, $\beta$ ) 对模型效果的影响, 根据环境变量对模型的贡献率及刀切法分析结 果, 选取重要性高的变量组合重新调整 $\beta$ 值建模 ${ }^{[37]}$.

1.2.3 福寿螺潜在分布区等级划分 依据 MaxEnt 模型预测的福寿螺分布的适宜程度, 选用 ArcGIS 软件中的 Reclass 工具, 对福寿螺的潜在分布区等级进行划分. 其中, 自然分割法 (Jenks) 的结果最接近真实的福寿螺 分布情况, 将其划分为 4 个适生等级 (无分布区、低分布区、中分布区和高分布区). 为了更加直观地展示预 测结果, 叠加中国国界、省界和中国三级河流以上的数据要素 (数据来源于国家基础地理信息中心网站 http ://nfgis.nsdi.gov.cn/.).

\section{2 结果与分析}

\section{1 模型预测准确性}

本研究基于 6 个环境变量建模的最优模型中, 训练数据 AUC 平均值为 0.981 (图 1), 测试数据 AUC 平 均值为 0.975 (95\% 置信区间: 0.948 0.993). 模型中测试数据平均值大于 0.97 , 说明 Maxent 模型预测结果精 确度高, 较好地预测了适宜福寿螺生活的潜在分布区域.

\section{2 影响福寿螺分布的环境因子分析}

通过空间自相关分析选取主要环境变量 (表 1 ) 构建最优模型,分析结果显示:年均温、等温性、温度季节 性变化标准差、年均降水量、降水量季节性变异系数以及海拔对福寿螺的潜在分布具有一定的影响. 使用刀 切法分析这 6 个主要环境变量对预测结果影响的贡献率, 由图 2 可以看出, 等温性对预测结果影响最小, 贡 献率为 $7.41 \%$, 贡献率最大的环境变量是年均温, 为 $25.78 \%$, 对福寿螺种群的分布和扩散起重要作用.

\section{3 福寿螺在我国的扩散动态}

福寿螺在中国的历史分布动态 (图 3) 表明,1980s 福寿螺被引进广东、福建、浙江、湖南、重庆、云南和台 湾等省, 并以引进地区为中心, 向四周扩散, 扩散速度为 $5.6 \mathrm{~km} / \mathrm{a} ; 1990 \mathrm{~s}$ 以后福寿螺被携带至各地, 辽宁省 朝阳市、甘肃省兰州市、河北邯単市虽有福寿螺养殖报道, 但由于地理气候因素无法定殖 ${ }^{[38-39]}$, 因此在计算 扩散速度时并未被纳人. 相对 $1980 \mathrm{~s}$, 其扩散速度增加到 $7.6 \mathrm{~km} / \mathrm{a}$, 扩散方向呈扇形逐渐向内陆延伸;2000 年 以后, 湖南和湖北的中南部、四川南部、浙江中部和北部、福建东南部地区均有广泛分布, 而江西省、广东省、 广西省大部分地区已经成为福寿螺主要集中区域, 其扩散速度达到 $7.04 \mathrm{~km} / \mathrm{a}$, 并且福寿螺在不同年代以不 同的扩散速度在我国境内扩散. 


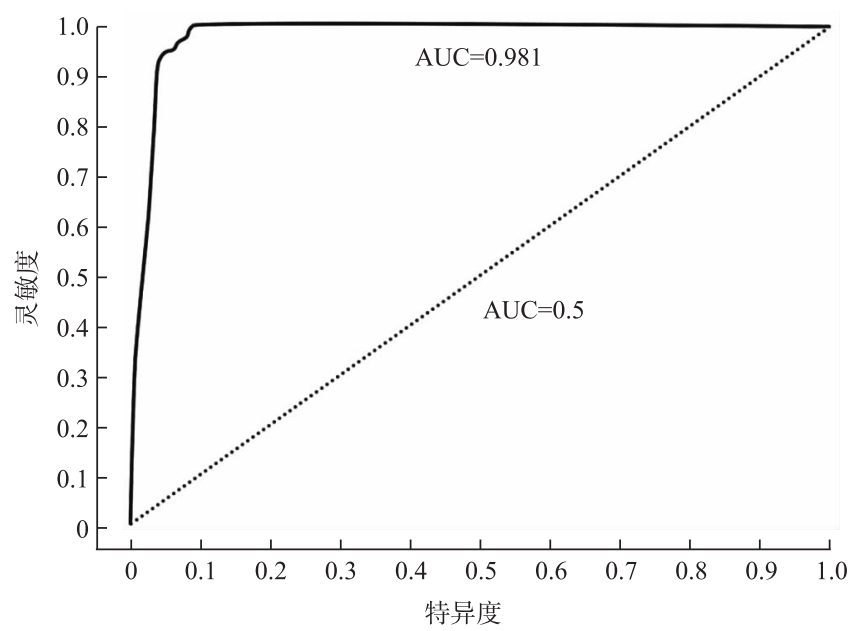

图 1 福寿螺预测结果的 AUC 曲线

Fig.1 AUC result of the prediction process of Pomacea spp.

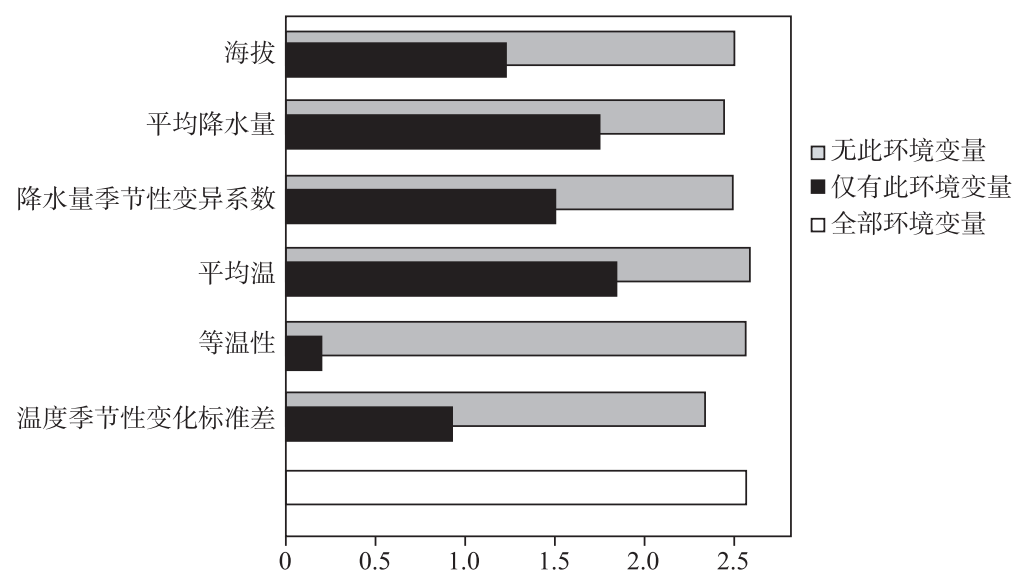

图 2 基于 Maxent 模型中刀切法的环境变量贡献率

Fig.2 Results of Jackknife test in Maxent model

\section{4 福寿螺在中国的分布预测}

基于 Maxent 模型对福寿螺在中国的潜在分布进行了预测. 福寿螺主要分布在长江流域及其以南的地 区, 其中浙江、福建、江西、广东、广西、海南和台湾等省已成为福寿螺潜在的高分布区,其危害十分严重; 而 上海、湖北、湖南、四川、西藏、贵州、重庆和云南虽为中分布区,但也具有较高的潜在暴发风险(图 4).

\section{3 讨论}

福寿螺在 $1990 \mathrm{~s}$ 被弃养至野外, 之后就在我国南方许多省份定殖 ${ }^{[40]}$, 物种的生态需求与本地螺类相似, 较为稳定, 物种迁移能力是无限的, 生态位是保守的 ${ }^{[41]}$, 符合物种生态位模型预测的条件 ${ }^{[42]}$, 因此, 福寿螺 的分布数据可用作模型预测.

影响模型预测准确度的主要是物种地理分布数据和其分布的环境变量 ${ }^{[43]}$. 陈新美等 ${ }^{[44]}$ 利用不同样本 量分别对 Maxent 模型预测的 4 个物种实际分布进行比较研究, 指出随着样本量的增大, 预测结果则越来越 精确, 当增大到一定数量时 $(>500)$, 训练数据和检验数据的 AUC 则趋近相同, Maxent 模型预测的精度和稳 

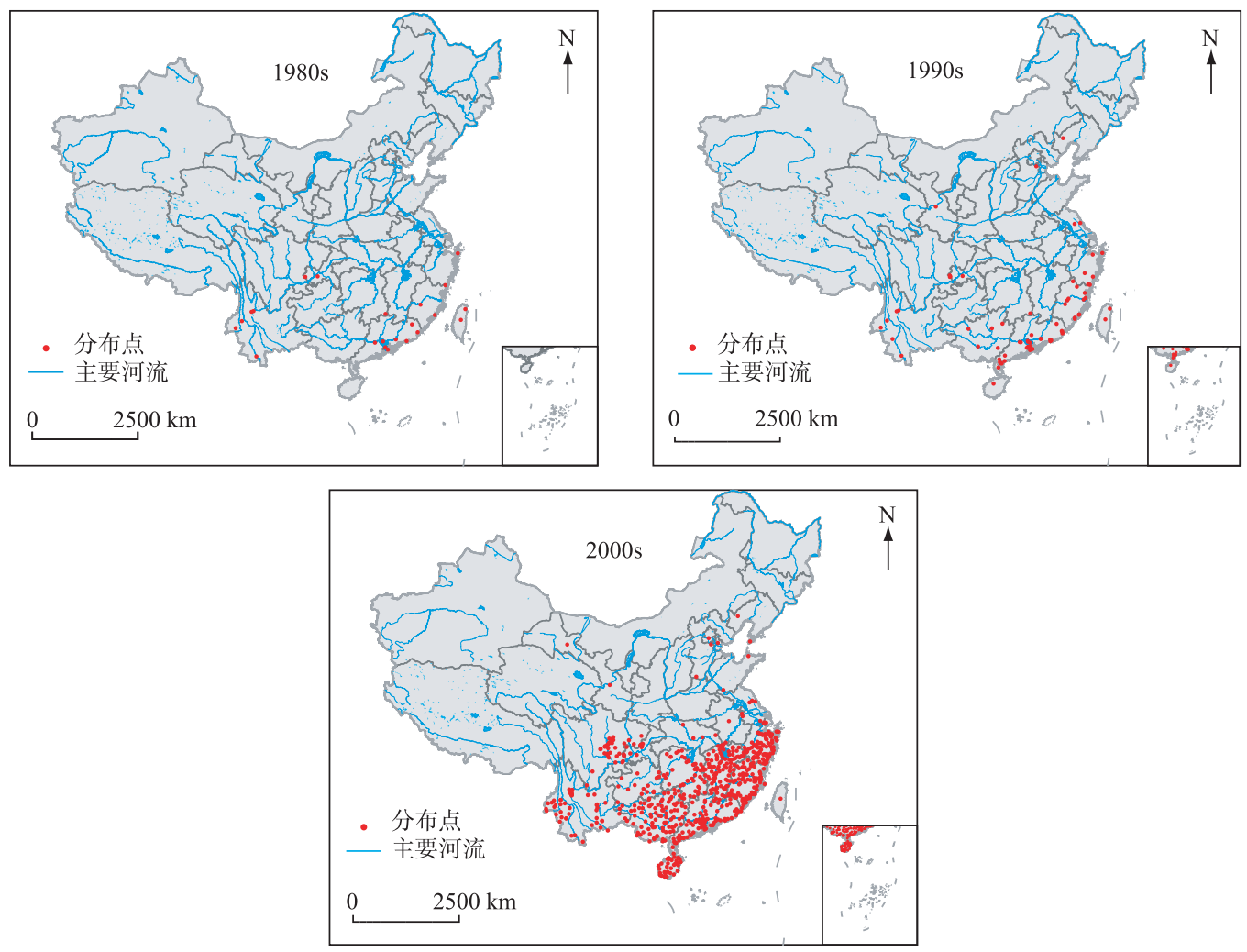

图 3 福寿螺在中国的历史扩散动态

Fig.3 Historical invasion and expansion process of Pomacea spp. in China

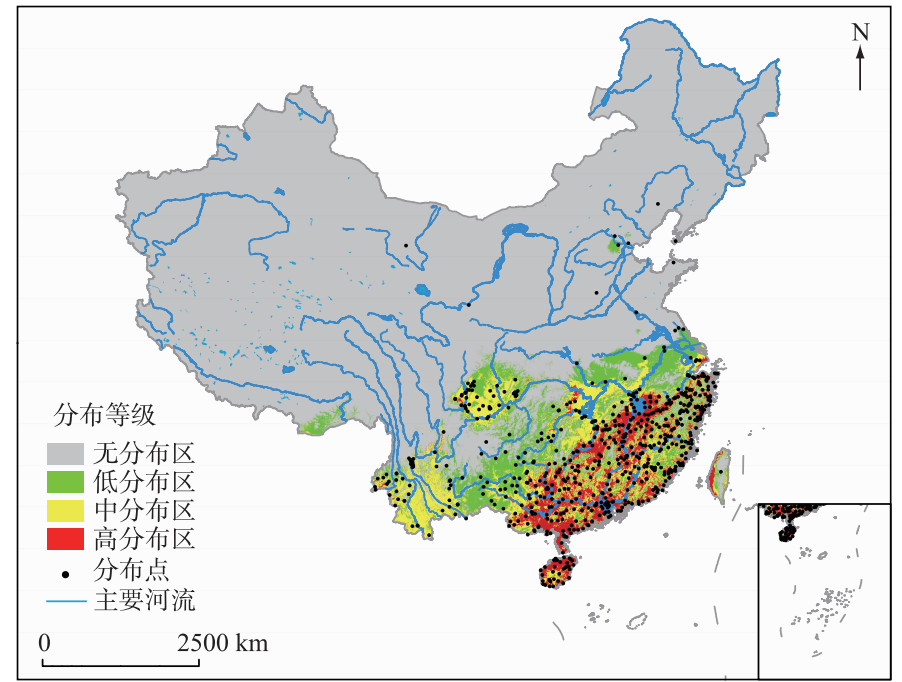

图 4 基于 Maxent 模型预测的 2017 年福寿螺潜在分布区

Fig.4 Distribution of Pomacea spp. predicted by the Maxent model in 2017 
定性将大大提高. Papes 等 ${ }^{[45]}$ 基于 Maxent 预测美国的人侵物种中国圆田螺,认为对于使用默认值 10000 的 背景样本量, 采集的样本分布数据不能小于背景样本量两个数量级 (即应>100), 收集的有效数据越多对模 型训练越有利. 张海涛等 ${ }^{[19]}$ 搜集了 337 条样本分布数据, 结合 19 个环境变量对福寿螺的潜在适生区进行预 测, 而本文收集的福寿螺分布数据为 683 条, 远高于最低样本量, 并通过空间自相关分析后笁选出 6 个主要 环境变量构建最佳模型, 预测结果大部分与张海涛等的研究结果一致, 其中福寿螺在广东、广西、湖南、浙江 和福建的沿海地区危害比较严重, 本文还补充了江西省和云南省, 这两个省份的部分地区达到了福寿螺高 分布等级, 这些地区的水系发达, 具有很高的扩散风险. 相关研究表明, 水体环境因子变化能影响福寿螺分 布 ${ }^{[46]}$. 野外调查也发现, 福寿螺在静水水体, 如稻田、藕田、池塘以及缓流的河流密度较大, 而大型河流、湖 泊和水库则密度小或未发现. 目前缺乏水体环境因子变量、水体底质类型等影响福寿螺分布的高分辨率环 境图层, 有可能规避了起决定性作用的环境变量, 但相关研究显示, 在大尺度上, 物种分布主要受气候环境 变量的影响, 仅在小尺度下再考虑水质理化因子造成的影响 ${ }^{[47]}$. 本研究构建的最优模型, 证明了基于生物 气候数据的模型能较好地反映福寿螺的潜在地理分布,影响福寿螺分布的主要环境变量是年均温.

福寿螺自引入中国以来, 经历了潜伏期和扩散期两个阶段. 根据中国外来入侵生物数据库 (http://www. chinaias.cn)、中国寄生虫种质资源共享服务平台 (http://www.tdrc.org.cn)、实地调查和文献资料获得的福寿 螺分布数据重建福寿螺的历史分布动态 (图 3), 在 1980s 初, 福寿螺作为养殖品种被引进台湾和广东省, 这 是福寿螺在中国的首次入侵, 周围的沿海地区和川渝地区也很快出现了福寿螺养殖的报道 ${ }^{[48]}$, 此阶段的福 寿螺仅有少数个体逃逸到野外, 并未建立种群, 此时处于潜伏期; 直至 1990s 初期, 福寿螺经在各个省市之间 交错引人,其分布区域逐渐向内陆延伸, 如江苏、江西、湖南、四川、贵州、广西、海南、河北、辽宁等省均报道 有福寿螺的分布. 由于其食味不佳, 市场供大于求, 大量福寿螺被养殖户遗弃至野外 ${ }^{[49]}$, 而后随水流、灌溉 流人江河、溪流、农田、池塘. 加之福寿螺食性广, 耐受性强, 生长繁殖快等因素, 在野外迅速建立了种群; 1990s 中期, 许多地区不仅出现了福寿螺危害经济作物的现象 ${ }^{[50]}$, 而且它还侵占了本地螺类的生态位, 对农 业经济和生态系统多样性带来极大的破坏, 此外, 其自身还携带有广州管圆线虫, 对人类造成严重威胁, 这 些因素使得政府逐渐重视并实施一系列治理措施. 2000 年后福寿螺在南方许多省市泛滥成灾. 尽管政府加 强一系列防治措施, 一定程度上抑制了其增长进程, 但效果不显著. 从福寿螺不同时期的分布 (图 3) 可知, 随着人为因素、水系流向的助力和全球变暖等环境因素的影响, 福寿螺在我国逐步由东南部沿海向中部和 西部扩散蔓延, 且具有向北扩散的趋势 (图 3). 从福寿螺的扩散速度来看, 1980-2010 年福寿螺在中国南部 的扩散速度在持续增长, 目前仍处在扩散阶段.

基于 Maxent 模型对福寿螺在我国扩散的预测结果, 我们认为适合福寿螺生存的潜在分布区主要位于南 方大部分地区, 具有向北扩散的趋势. 尽管在辽宁省朝阳市、北京市、河北省 (石家庄市、邯郸市)、甘肃省兰 州市均报道有福寿螺分布, 但由于福寿螺很难随长江水系自然扩散至北方, 且北方冬季气候寒冷, 福寿螺在 $6^{\circ} \mathrm{C}$ 下即不能存活, 因此福寿螺不具备在北方成功越冬的能力, 很难在北方建立种群 ${ }^{[51-52]}$. 随着人为有意或 无意的携带, 以及地理气候、水系流向等因素产生的自然扩散,给福寿螺在中国南部的快速扩散提供了人侵 条件, 对于适生等级为低适生区的地区, 例如贵州省、湖北省, 福寿螺可能会进一步人侵和扩散, 可能形成全 面人侵的分布格局. 为避免我国农业经济和生态系统遭受严重破坏, 必须重视福寿螺灾害的防治, 严格监测 福寿螺的人侵趋势. 对已受灾害的地区采取有效的防治措施, 以及对福寿螺尚未人侵但具有高度人侵风险 的地区进行早期预警监测, 从而控制福寿螺进一步扩散.

\section{4 参考文献}

[ 1 ] Board MEA. Millenium ecosystem assessment-ecosystems and human well-being: wetlands and water synthesis. Physics Teacher, 2005, 34(9): 534.

[ 2 ] Xu HG, Ding H, Li M et al. The distribution and economic losses of alien species invasion to China. Biological Invasions, $2006,8(7): 1495-1500$

[ 3 ] Lowe S, Browne M, Boudjelas S et al. 100 of the world's worst invasive alien species: a selection from the global invasive species database. Invasive Species Specialists Group, 2000, 12: 1-12.

[ 4 ] Karraker NE, Dudgeon D. Invasive apple snails (Pomacea canaliculata) are predators of amphibians in South China. Bio- 
logical Invasions, 2014, 16(9) : 1785-1789.

[ 5 ] Halwart M. The golden apple snail Pomacea canaliculata in Asian rice farming systems: Present impact and future threat. International Journal of Pest Management, 1994, 40(2) : 199-206.

[ 6 ] Zhang Y, Lv S, Yang K et al. The first national survey on natural nidi of Angiostrongylus cantonensis in China. Chinese Journal of Parasitology and Parasitic Diseases , 2009, 27(6): 508-512. [张仪, 吕山, 杨坤等. 我国广州管圆线虫自 然疫源地分布首次调查. 中国寄生虫学与寄生虫病杂志, 2009, 27(6) : 508-512.]

[ 7 ] China Environmental Protection Administration ed. China's first list of invasive alien species. Journal of the State Council of the People's Republic of China, 2003, 23: 41-46. [中国环境保护总局. 中国第一批外来人侵物种名单. 中华人民共 和国国务院公报, 2003, 23: 41-46.]

[ 8 ] Cazzaniga NJ. Sexual dimorphism in Pomacea canaliculata (Gastropoda: Ampullariidae). Veliger, 1990, 33 (4): 384-388.

[ 9 ] Ye M, Fan TQ, Chen L et al. Comparative study on morphologcial of radula of Pomacea canaliculata, P. maculata and Cipangopaludina chinensis. Chinese Journal of Zoology, 2017, 52 (1) : 97-107. [叶苗, 㚞天骐, 陈炼. 两种福寿螺与中 国圆田螺齿舌的形态学特征比较. 动物学杂志, 2017, 52(1): 97-107.]

[10] Zhou W, Dao XQ, Deng CM et al. Study on the reproduction and growth of Pomacea canaliculata. Journal of Anhui Agricultural Sciences, 2015, 43(11): 134-135, 138. [周外, 刀学琼, 邓才明等. 福寿螺的繁殖与生长研究. 安徽农业科 学, 2015, 43(11): 134-135, 138.]

[11] Tamburi NE, Martín PR. Effects of food availability on reproductive output, offspring quality and reproductive efficiency in the apple snail Pomacea canaliculata. Biological Invasions, 2011, 13(10) : 2351-2360.

[12] Wang YH. Apple snails (Ampullorum crossean) breeding and ecological characteristics. Chinese Journal of Zoology, 1988, 23(1) : 4-6. [王彝豪. 福寿螺的养殖和生态特点. 动物学杂志, 1988, 23(1) : 4-6. ]

[13] Guo J, Zhang JE. Status, problems and countermeasures in biological control of Pomacea canaliculata. Chinese Journal of Ecology, 2015, 34(10) : 2943-2950. [ 郭靖, 章家恩. 福寿螺的生物防治现状, 问题与对策. 生态学杂志, 2015, 34 (10) : 2943-2950.]

[14] Wei JQ, Mo JC, Hong WY et al. Progress in integrated control of Pomacea canaliculata, intermediate host of Angiostrongylus cantonensis. Chinese Journal of Vector Biology and Control, 2010, 21 (5) : 512-514. [ 尉吉乾, 莫建初, 洪文英等. 广州管圆线虫中间宿主福寿螺的综合防治研究进展. 中国媒介生物学及控制杂志, 2010, 21(5): 512-514.]

[15] Liu YF, Li F, Li YF et al. The distribution situation, damage and early warning on diffusion risk of golden apple snail, Pomaces canaliculata Lamark in Hunan. Acta Hydrobiologica Sinica, 2011, 35(6): 1067-1071. [刘雨芳, 李菲, 李玉峰 等. 福寿螺在湖南的分布现状、危害与扩散风险预警. 水生生物学报, 2011, 35(6): 1067-1071.]

[16] He MQ, Luo MZ, Zhang JE en al. Damage status and control countermeasure of Pomacea canalicultata in Guangdong. Guizhou Agriculture Sciences, 2011, 39(1) : 100-103. [何铭谦, 罗明珠, 章家恩等. 广东福寿螺爆发危害状况调查 及防治对策. 贵州农业科学, 2011, 39(1): 100-103]

[17] Zhu GP, Liu GQ, Bu WJ et al. Ecological niche modeling and its applications in biodiversity conservation. Biodiversity Science, 2013, 21 (1) : 90-98. [ 朱耿平, 刘国卿, 卜文俊等. 生态位模型的基本原理及其在生物多样性保护中的应 用. 生物多样性, 2013, 21(1): 90-98.]

[18] Elith J, Graham CH, Anderson RP et al. Novel methods improve prediction of species' distributions from occurrence data. Ecography, 2006, 29(2): 129-151.

[19] Zhang HT, Luo D, Mou XD et al. Predicting the potential suitable distribution area of the apple snail Pomacea canaliculata in China based on multiple ecological niche models. Chinese Journal of Applied Ecology, 2016, 27 (4) : 1277-1284. [张海涛, 罗渡, 牟希东等. 应用多个生态位模型预测福寿螺在中国的潜在适生区. 应用生态学报, 2016, 27(4): 1277-1284.]

[20] Habib MR, Guo YH, Lv S et al. Predicting the spatial distribution of Biomphalaria straminea, a potential intermediate host for Schistosoma mansoni, in China. Geospatial Health, 2016, 11(3) : 375-383.

[21] Li XH, Gao ST, Gu WB et al. Species identification of freshwater snail Planorbella trivolvis and analysis of its potential distribution. Chinese Journal of Parasitology and Parasitic Diseases, 2015, 27(3): 268-272. [李晓恒, 高世同, 顾文彪等. 淡水螺类三旋丽卷螺的物种鉴定及其潜在适生区分析. 中国血吸虫病防治杂志, 2015, 27(3) : 268-272.]

[22] Qi GJ, Gao Y, Huang DC et al. Historical invasion, expansion process and the potential geographic distributions for the 
rice water weevil, Lissorhoptrus oryzophilus in China based on Maxent. Acta Phytophylacica Sinica, 2012, 39(2): 129136. [齐国君, 高燕, 黄德超等. 基于 Maxent 的稻水象甲在中国的人侵扩散动态及适生性分析. 植物保护学报, $2012,39(2): 129-136$.

[23] Han YY, Wang Y, Xiang Y et al. Prediction of potential distribution of Burasaphelenchus xylophilus in China based on Maxent ecological niche model. Journal of Nanjing Forestry University, 2015, 39(1): 6-10. [韩阳阳, 王炎, 项杨等. 基 于 Maxent 生态位模型的松材线虫在中国的适生区预测分析. 南京林业大学学报, 2015, 39(1) : 6-10.]

[24] Zhang XA, Sui XY, Lv Z et al. A prediction of the global habitat of two invasive fishes (Pseudorasbora parva and Carassius auratus) from East Asia using Maxent. Biodiversity Science, 2014, 22(2) : 182-188. [张熙謷, 隋晓云, 吕植等. 基于 Maxent 的两种人侵性鱼类 (麦穗鱼和鲫) 的全球适生区预测. 生物多样性, 2014, 22(2) : 182-188.]

[25] Li MY, Ju YW, Sunil K et al. Modeling potential habitat for alien species of Dressena polymorpha in the continental USA. Acta Ecologica Sinica, 2008, 28(9) : 4253-4258. [李明阳, 巨云为, Sunil K 等. 美国大陆外来人侵物种斑马纹贻贝 (Dreissena polymorpha) 潜在生境预测模型. 生态学报, 2008, 28(9) : 4253-4258.]

[26] Byers JE, Mcdowell WG, Dodd SR et al. Climate and $\mathrm{pH}$ predict the potential range of the invasive apple snail (Pomacea insularum) in the southeastern United States. PLoS One, 2013, 8(2) : e56812.

[27] Lin W, Han XM, Xu Y et al. Introduction to the web “bio-invasion in China”. Journal of Beijing Normal University: Natural Science, 2006, 42(2): 189-194. [林雯, 韩雪梅, 徐岩等. 介绍中国生物人侵网. 北京师范大学学报: 自然科 学版, 2006, 42(2): 189-194.]

[28] Lv S, Zhang Y, Liu HX et al. Invasive snails and an emerging infectious disease: results from the first national survey on Angiostrongylus cantonensis in China. PLoS Neglected Tropical Diseases, 2009, 3(2): e368.

[29] Wang R, Wang YZ. Invasion dynamics and potential spread of the invasive alien plant species Ageratina adenophora (Asteraceae) in China. Diversity \& Distributions, 2010, 12(4) : 397-408.

[30] Li WJ, Wang ZJ. A wintering habitat model for red crown crane. Chinese Journal of Applied Ecology, 2000, 11(6) : 839842. [李文军, 王子健. 丹顶鹤越冬栖息地数学模型的建立. 应用生态学报, 2000, 11(6) : 839-842.]

[31] Hengeveld R. Dynamics of biological invasions. Biometrics, 1989, 47(1) : 1-142.

[32] Shigesada N. Biological invasions: theory and practice. American Journal of Human Biology, 1998, 10(5) : 683-684.

[33] Andow DA, Kareiva PM, Levin SA et al. Spread of invading organisms: patterns of spread. In: Kim KC ed. Evolution of insect pests: The pattern of variations. New York: Wiley, 1993: 219-242.

[34] Wang R, Wang YZ. Invasion dynamics and potential spread of the invasive alien plant species Ageratina adenophora (Asteraceae) in China. Diversity and Distributions, 2006, 12(4) : 397-408.

[35] Hanley JA, Mcneil BJ. The meaning and use of the area under a receiver operating characteristic curve (ROC). Radiolo$g y, 1982,143(1): 29-36$.

[36] Wang YS, Xie BY, Wan FH et al. Application of ROC curve analysis in evaluating the performance of alien species' potential distribution models. Biodiversity Science, 2007, 15(4): 365-372. [王运生, 谢丙炎, 万方浩等. ROC 曲线分析在 评价人侵物种分布模型中的应用. 生物多样性, 2007, 15(4) : 365-372.]

[37] Warren DL, Wright AN, Seifert SN et al. Incorporating model complexity and spatial sampling bias into ecological niche models of climate change risks faced by 90 California vertebrate species of concern. Diversity \& Distributions, 2014,20 (3) : 334-343.

[38] Yu GJ, Cheng XZ. Observation on the breeding and living habits of Ampullaria gigas spix. Chinese Journal of zoology, 1993, 28(3) : 48-50. [于国江, 程秀珍. 大瓶螺养殖与生活习性的观察. 动物学杂志, 1993, 28(3) : 48-50.]

[39] Yang YT, Tang YQ. Studies on the introduced apple snail breed (Ampullaria gigas) in Jiuquan, Gansu. Journal of Northwest University for Nationalities: Natural Science, 1992, (1): 71-73. [杨友桃, 唐迎秋. 酒泉市引进福寿螺的养殖研 究. 西北民族大学学报: 自然科学版, 1992, (1): 71-73.]

[40] Li XH, Hu YC, Song HM et al. Invasion and monitoring methods of Pomacea canaliculata for in China. Chinese Agricultural Science Bulletin, 2009, 25 (14) : 229-232. [李小慧, 胡隐昌, 宋红梅等. 中国福寿螺的人侵现状及防治方法研究 进展. 中国农学通报, 2009, 25(14): 229-232.]

[41] Peterson AT. Ecological niche conservatism: a time-structured review of evidence. Journal of Biogeography, 2011,38 (5) : 817-827.

[42] Wiens JJ, Graham CH. Niche conservatism: integrating evolution, ecology, and conservation biology. Annual Review of E- 
cology Evolution \& Systematics, 2005, 36: 519-539.

[43] Cai JY, Zhang MM, Su HJ et al. Application of ecological niche models for selection of species habitat. Journal of Economic Animal , 2014, 18(1) : 47-52. [蔡静芸, 张明明, 粟海军等. 生态位模型在物种生境选择中的应用研究. 经济动 物学报, $2014, \mathbf{1 8}(1): 47-52$.]

[44] Chen XM, Lei YC, Zhang XQ et al. Effects of sample sizes on accuracy and stability of maximum entropy model in predicting species distribution. Scientia Silvae Sinicae, 2012, 48(1) : 53-59. [ 陈新美, 雷渊才, 张雄清等. 样本量对 Maxent 模型预测物种分布精度和稳定性的影响. 林业科学, 2012, 48(1) : 53-59.]

[45] Papes M, Havel JE, Vander Zanden MJ. Using maximum entropy to predict the potential distribution of an invasive freshwater snail. Freshwater Biology, 2016, 61: 457-471.

[46] Guo GG, Zhang SM, Fan W et al. Comparative research on habitat suitability index model of albacore tuna (Thunnus alalunga) based on surface and thermocline environmental variables in the South Pacific Ocean. Acta Oceanologica Sinica, 2016, 38(10) : 44-51. [ 郭刚刚, 张胜茂, 㚞伟等. 基于表层及温跃层环境变量的南太平洋长鳍金枪鱼栖息地适应 性指数模型比较. 海洋学报, 2016, 38(10): 44-51.]

[47] Zhu GP, Liu Q, Gao YB. Improving ecological niche model transferability to predict the potential distribution of invasive exotic species. Biodiversity Science, 2014, 22(2): 223-230. [朱耿平, 刘强, 高玉葆. 提高生态位模型转移能力来模 拟人侵物种的潜在分布. 生物多样性, 2014, 22(2): 223-230.]

[48] Yu XP, He TJ, Li ZF et al. Occurrence of golden apple snail Pomacea canaliculata (Lamarck) in paddy fields and its management. Acta Agriculturae Zhejiangensis, 2001, 13(5): 247-252. [俞晓平, 和田节, 李中方等. 稻田福寿螺的发 生和治理. 浙江农业学报, 2001, 13(5): 247-252.]

[49] Yang YX, Hu YC, Li XH et al. Historical invasion, expansion process and harm investigation of Pomacea canaliculata in China. Chinese Agricultural Science Bulletin, 2010, 26(5): 245-250. [杨叶欣, 胡隐昌, 李小慧等. 福寿螺在中国的 人侵历史、扩散规律和危害的调查分析. 中国农学通报, 2010, 26(5): 245-250.]

[50] Zhao BL, Zhang JE, Dai XY et al. Effects of Pomacea canaliculata on aquatic macrophyte community in paddy fields. Acta Ecologica Sinica, 34(4) : 907-915. [赵本良, 章家恩, 戴晓燕等. 福寿螺对稻田水生植物群落实验的影响. 生态学 报, 2014, 34(4): 907-915.]

[51] Wada T, Matsukura K. Linkage of cold hardiness with desiccation tolerance in the invasive freshwater apple snail, Pomacea canaliculata (Caenogastroppoda: Ampullariidae). Journal of Molluscan Studies, 2011, 77: 149-153.

[52] Deaton LE, Schmidt W, Leblanc B et al. Physiology of the invasive apple snail Pomacea maculata: tolerance to low temperatures. Journal of Shellfish Research, 2007, 35(1) : 207-210. 